\title{
Hacia una comprensión de las relaciones de interdependencia en el ecosistema de aprendizaje
}

\section{Towards an understanding of interdependent relationships in the learning ecosystem}

\author{
William Rodrigo Avendaño Castro', \\ Audin Aloiso Gamboa Suárez², \\ Raúl Prada-Núñez ${ }^{3}$ \\ UFPS
}

\section{RESUMEN}

El objetivo de la investigación fue describir las relaciones de interdependencia positivas que se contextualizan y se establecen en el ecosistema de aprendizaje, por parte de los docentes del contexto universitario que pertenecen a una red de matemática. La investigación se enmarcó en

$\overline{1}$ Doctor en Ciencias Sociales y Humanas por la Pontificia Universidad Javeriana. Docente investigador de la Universidad Francisco de Paula Santander. Correo electrónico: williamavendano@ufps.edu.co. Orcid: 0000-00027510-8222.

2 Doctor en Ciencias de la Educación por la Universidad de Cartagena (Colombia). Docente investigador de la Universidad Francisco de Paula Santander. Correo electrónico: audingamboa@ufps.edu.co. Orcid: 0000-00019755-6408.

3 Magíster en Ingeniería de Análisis de Datos, Mejora de Procesos y Toma de Decisiones por la Universidad Politécnica de Valencia (España). Docente investigador de la Universidad Francisco de Paula Santander. Correo electrónico: raulprada@ufps.edu.co. Orcid: 0000-0001-61451786. un enfoque mixto, de tipo aplicada y descriptiva. La muestra estuvo compuesta por veinticinco (25) docentes del contexto universitario. Los instrumentos utilizados fueron la encuesta y la entrevista semiestructurada. El estudio mostró que la relación de interdependencia en la presencia cognitiva facilita el acceso a los recursos y materiales de aprendizaje, así como la interdependencia en la interacción con los docentes, las redes y la relación entre pares resulta de una integración del espacio de colaboración y cooperación. Se concluyó que el ecosistema de aprendizaje permite establecer relaciones positivas de interdependencia para la transmisión, producción de conocimiento y nodos de saberes. 


\section{PALABRAS CLAVE:}

relaciones, interdependencia positiva, ecosistema de aprendizaje

\section{ABSTRACT}

The objective of the research was to describe the positive interdependence relationships that are contextualized and established in the learning ecosystem, by professors from the university context who belong to a mathematics network. The research was framed in a mixed approach, of applied and descriptive type. The sample consisted of twenty-five (25) professors from the university context. The instruments used were the survey and the semi-structured interview. The study showed that the relationship of interdependence in cognitive presence facilitates access to resources and learning materials, as well as interdependence in the interaction with educators, networks and the relationship between peers results from an integration of the collaboration space and cooperation. It was concluded that the learning ecosystem allows establishing positive relationships of interdependence for the transmission, production of knowledge and nodes of knowledge.

\section{KEYWORDS:}

relationships, positive interdependence, learning ecosystem

\section{INTRODUCCIÓN}

El proceso de enseñanza-aprendizaje basado en entornos personales de aprendizaje (en inglés, Personal Learning Environment, PLE) ha transitado hacia un ecosistema pedagógico (Castañeda y Adell, 2013), en el cual interaccionan estudiantes y docentes que se apoyan herramientas, actividades, fuentes de información y nodos de conexiones de forma recurrente (Calle y Sánchez, 2017). Es decir, hay presencia de personas, dispositivos, servicios y recursos de preferencia personal que deja en evidencia una relación de interaccióninterdependencia en "la presencia social" (Garrison et al., 2000).

Por otro lado, el concepto de ecosistema (sistema biótico/abiótico), es utilizado por diversos autores para sustentar su propia definición de ecosistema (García-Peñalvo, 2018). Asimismo, Frielick, (2004) señala que aflora en la literatura conceptos sobre "... las redes como ecosistemas..." (p. 238). Así como, la adopción del concepto de "ecosistema de aprendizaje digital" (Laanpere et al., 2014, p. 422). También, Siemens (2005), asume la creación de significados y relaciones públicas en entornos de software social a través de procesos conectivos a la formación de nuevas ecologías de conocimiento y culturas de aprendizaje. En el marco del conectivismo, este autor, adopta un enfoque inclinado hacia el conocimiento, los significados, las comunidades, las redes y sus espacios, es decir, hacia los ecosistemas, que se puede entender como dar forma a una interdependencia en la gestión del conocimiento.

Para Guetl y Chang (2008), un ecosistema de aprendizaje es una definición abstracta que clasifica los componentes bióticos y abióticos y, todas sus interrelaciones (de interdependencia) entre los estudiantes y docentes, incluso, con proveedores de contenido, diseñadores instruccionales y expertos pedagógicos, siendo estos la parte viva del ecosistema de aprendizaje. Las unidades abióticas, refiere a las partes no vivas, que incluyen los medios de formación (contenidos, materiales y aspectos pedagógicos) y tecnología. Como se puede apreciar, las relaciones e interacciones relacionadas con el flujo de información, así como la transferencia y transformación del conocimiento se manifiestan por la influencia tanto interna como externa de los actores en el dominio del aprendizaje, o sea, constituyen un espacio de encuentros e interacciones en el qué, y a través de los cuales, los sujetos coorganizados en el ecosistema del contexto digital se apoyan en sus procesos de 
aprendizaje (Gómez-Valderrama et al., 2020). Además, las comprensiones priorizan a las formas activas, colaborativas, (Prada et al., 2019), con vínculos conectivos que destaca "... el acto de aprender <al> crear una red externa de nodos, donde se conectan y forman fuentes de información y conocimiento..." (Siemens, 2006, p. 29).

De ahí, los vínculos entre los individuos, es lo que Johnson y Johnson (1999) denomina interdependencia social. Por ello, las relaciones de interdependencia pueden ser positivas, ya que se establecen una interrelación de cooperación en el contexto social. Además, de ser un vehículo que fomenta la participación y ayuda mutua en el proceso de enseñanza-aprendizaje. En este punto, el término interdependencia positiva se utiliza para definir aquella situación en la que el aprendizaje de los diferentes componentes del grupo depende de las acciones que realice cada uno de los miembros del equipo. En ese sentido, Jonhson y Jonhson (1989) refieren que la interdependencia positiva está vinculada cooperativamente para alcanzar las metas del grupo, siempre y cuando las personas perciben que pueden alcanzar sus propios objetivos junto a las de otras personas. Por ello, incide en la interacción, en el equipo de trabajo y en el rendimiento individual. De este modo, expande el logro particular al beneficio colectivo por medio de las acciones de interés mutuo que sustituyen a las propias en el logro de metas.

De acuerdo con Jonhson et al., (2008), los tipos de interdependencia positiva son: a) La interdependencia positiva respecto de las metas, que tiene el efecto de unir a los miembros del grupo en torno a un objetivo en común. Esta estrategia tiene diversas limitaciones, según Cuadrado et al., (2014), pues “...no resulta fácil ajustar la carga de trabajo a las horas previstas, porque hay tareas que algunos alumnos hacen en menos tiempo que otros, $y, \ldots$ alumnos dispuestos a dedicar más horas... para hacer su parte y la de los demás..." (p. 364); b) La interdependencia respecto de los recursos, que significa que se proporciona sólo una parte de la información, los materiales didácticos u otros elementos necesarios para realizar una tarea, de modo que los miembros tengan que combinar sus recursos a efecto de lograr sus objetivos; c) la interdependencia respecto de los premios, que se reconoce a través de la observación el esfuerzo de los estudiantes por aprender y promover el aprendizaje de sus compañeros. También, la declaración del esfuerzo y rendimiento mutuo crea responsabilidad ante el beneficio del grupo; d) Interdependencia de los roles, que detallan el trabajo de cada uno, en las actividades que lleva adelante el grupo para efectuar un trabajo conjunto. Según Cuadrado et al., (2014), los roles pueden ser funcionales y asigna a los miembros del grupo roles como: secretario, el portavoz, el director, entre otros.

De lo anterior, se observa que la interdependencia resalta la responsabilidad mutua en el proceso de aprendizaje y transforma una actividad grupal en una actividad de mutualidad, donde se conectan los miembros del grupo. Dentro de esta perspectiva, la interdependencia positiva es una relación en la que todos ganan y viene dada por la comunicación, colaboración, y el trabajo grupal cooperativo (Johnson y Johnson, 2008). Por ello," la autoridad del docente se comparte con los estudiantes." (Gutiérrez y Becerra, 2014, p. 51), bajo un conjunto de indicadores, que circunscriben una ayuda $y$ asistencia mutua, intercambio de los recursos necesarios, comunicación eficaz, influencia mutua, confianza y manejo constructivo del conflicto. Se entiende que el docente fomenta una interacción promotora, que incita y facilita los esfuerzos de los demás para completar tareas, y lograr, producir o alcanzar las metas del grupo.

Por otro lado, en los entornos y redes personales de aprendizaje se desarrolla interdependencia 
positiva, y, con ella, la interacción, la contribución, la comunicación y la colaboración del grupo, la cual se debe a la integración (Marín et al., 2014). Al considerar lo anterior, las relaciones de interdependencia positiva en el ecosistema de aprendizaje se deben dar a partir de la: a) Comunicación, desde el ecosistema de aprendizaje "implica entender la construcción del conocimiento como un acto social y colectivo" (Pérez, 2009, p. 41), que propicia el desarrollo de relaciones interpersonales que favorecen el aprendizaje y la cohesión del grupo, a través del establecimiento de objetivos comunes y redes de aprendizaje, por tanto, se presenta una interdependencia. Ello se da por la "sincronía en la comunicación de equipos virtuales de trabajo. Si la interdependencia asegura la unión de los equipos tradicionales, las tecnologías de la comunicación hacen lo propio... convirtiéndose en su medio principal de interacción." (Rico et al., 2006, p. 744); b) Cooperación, que se refiere a la responsabilidad individual, interacción promotora, habilidades sociales (Johnson y Johnson, 1989). Además, es un proceso comunicativo de construcción de significados, conjunto que, de manera estructurada, promueve la interacción entre iguales en línea, en torno a un objetivo o tarea educativa interdependiente (Casanova et al., 2009). De este modo, las relaciones de cooperación establecen interdependencia positiva entre los componentes del grupo que se apoya en la comunicación y la interacción como elementos esenciales. Es un compromiso de todos y cada uno de los miembros hacia la meta conjunta de aprendizaje del equipo (García y Suárez, 2011, p. 481); c) Colaboración, la que da cuenta de la participación y corresponsabilidad, se basa en el aporte de cada miembro al desarrollo cognitivo y sociocultural de los demás y, en consecuencia, aprende más de lo que aprendería por sí solo (Martínez, 2015, p. 23). Por ello, es un "proceso compartido, coordinado e interdependiente, en el cual los estudiantes trabajan juntos para alcanzar un objetivo común en un entorno virtual... facilitando la construcción conjunta de significados y un avance individual hacia niveles superiores de desarrollo" (Guitert y PérezMateo, 2013, p. 24-25).

A partir de estos argumentos, es viable examinar las relaciones de interdependencia en el ecosistema de aprendizaje, en un contexto universitario. Pues, la disponibilidad colectiva de saberes (nodos) basados en la web 2.0, hace que sea importante prestar atención, al mundo de los ecosistemas digitales y lo que significa administrar, usar y publicar información y conexiones (personas) que facilitan procesos de construcción colectiva de contenidos (Pérez, 2011), en un ecosistema de aprendizaje para conectar las interacciones de los actores. Que, no solo facilita el acceso a los recursos y materiales didácticos, la interdisciplinariedad, y la reciprocidad de habilidades, sino a las relaciones con docentes, redes y entre pares, a partir de la reflexión sobre la validez de las ideas y opiniones de manera autodeterminada (Castañeda y Adell, 2013).

Por consiguiente, se entiende que el participante al estar en función de esa relación pareciera triangular entre docente, conocimiento y sus pares. De esta manera, se requiere un cambio en las prácticas educativas de los actores universitarios, pues, las relaciones en el ecosistema de aprendizaje, de acuerdo con Morín (2001) conducen a una idea aparentemente paradójica: la de que, para ser independiente, es necesario ser dependiente; cuanto se quiere ganar independencia, es necesario pagarla mediante la dependencia. Por tanto, dicha dependencia forma interdependencia en las interacciones con el aprendizaje, la cuales puede ser: positiva, negativa y la ausencia de interdependencia (Johnson y Johnson, 2014). Siendo la positiva, la cooperación que se genera en el grupo, mientras que la negativa, trata sobre la competencia y efectos de poder dentro del mismo. Para contribuir a esto, se trabajó en las 
experiencias de docentes universitarios, para establecer las relaciones de interdependencia en el ecosistema de aprendizaje. Por ello, el objetivo consistió en describir las relaciones de interdependencia positivas que se contextualizan y se establecen en el ecosistema de aprendizaje, por parte de los docentes que pertenecen a una red de gestión del conocimiento en el área de matemática.

\section{MÉTODO}

El estudio se enmarcó en un enfoque mixto. De este modo, la propuesta reconoce que actualmente no se configura el ecosistema de aprendizaje para fomentar las relaciones de interdependencia positiva. Sobre esa base se pueden hacer las siguientes afirmaciones: a) Supuesto cualitativo 1: Una estructura que incorpora la presencia social, cognitiva y del docente motivará relaciones de interdependencia positiva, permitirá el logro de las metas y objetivos propuestos en el ecosistema de aprendizaje; b) Supuesto cualitativo 2: El ecosistema de aprendizaje con recursos abiertos favorece las relaciones de interdependencia positiva.

Es de tipo aplicada, pues "se caracteriza por el modo de analizar la realidad social, anteponiendo la inmediatez en la resolución de los problemas sociales...su descubrimiento se aplica para crear, desarrollar o mejorar estrategias..." (Cívicos y Hernández, 2007, p. 37-38). De este modo, la aplicación consistió en el diseño de un ecosistema de aprendizaje con recursos abiertos, y la puesta en marcha de una formación online promovido por la Red de Matemática Norte Santander, Cúcuta-Colombia (Hernández-Suárez, 2020), y en el marco de este, es un estudio descriptivo porque busca especificar propiedades, características y rasgos importantes.

La población estuvo constituida conformadas por 75 docentes que son parte activa en la
Red de Matemáticas de Norte de SantanderColombia. En cuanto a la muestra, la misma es no probabilística, dado que el requisito para la selección fue pertenecer al contexto universitario, de allí, la muestra quedó integrada por veinticinco (25) docentes, de los cuales el $60.0 \%$ (15) son de género femenino y el resto, sexo masculino (10). Un $30.0 \%$ son profesionales universitarios, un $68.0 \%$ son magister, y un $2.0 \%$ posee estudios doctorales. Finalmente, se escogieron seis (6) docentes que tuvieron en común participar de entrevistas.

Como procedimiento, el ecosistema de aprendizaje se basó en la propuesta de Guetl y Chang (2007), donde se establecen las partes interesadas del aprendizaje (unidades bióticas o participantes) y entorno personal de aprendizaje apoyado con recursos abiertos (unidades abióticas). En lo referente, a las relaciones de interdependencia positiva (Jonhson y Jonhson, 2008), se presentan las dimensiones de comunicación, cooperación y colaboración, de acuerdo con una propuesta pedagógica de una comunidad de indagación en línea en la que se forman las presencias tanto cognitiva, social como la docente (Garrison et al., 2000), fortalecidas con ideas conectivistas (Siemens, 2005). Dentro de la presencia docente está el diseño de materiales de aprendizaje, la mediación y la enseñanza en línea; es decir, la coordinación. La presencia cognitiva enfatiza la forma en que los participantes son capaces de construir significado, a través de una comunicación sostenida con eventos desencadenantes, exploratorios, integradores y resolutivos. La presencia social proyecta las particularidades personales de los participantes mediante la comunicación abierta.

Para la recolección de la información se utilizó una encuesta con preguntas cerradas, así como, una entrevista semiestructurada. EI cuestionario inicial, estuvo integrado 35 ítems de 5 alternativas de respuesta: Siempre (S), Casi 
Siempre (CS) Algunas Veces (AV), Casi Nunca (CN) y Nunca $(\mathrm{N})$ con la finalidad de recoger información las relaciones de interdependencia positiva que establecen los participantes del curso en el ecosistema de aprendizaje. Para la validez se solicitó la evaluación de expertos en la materia. De igual manera, se aplicó una prueba piloto para determinar la confiabilidad por Alpha de Cronbach, a partir de las dimensiones e indicadores estudiados $(\alpha=0.78)$. Mientras que la entrevista semiestructurada se llevó a cabo mediante un guion de 9 preguntas relacionadas con la interdependencia positiva.

El análisis se realizó mediante estadísticas descriptivas, con el uso de Excel, lo que permitió tener una comprensión de las relaciones de interdependencia positiva en el ecosistema de aprendizaje. De igual manera, se usó dos (2) herramientas complementarias: (1) los datos de archivo y las huellas de las actividades de aprendizaje en el ecosistema de aprendizaje. Éstas fueron principalmente estadísticas sobre conexiones, historia de discusiones, actividades de aprendizaje y, acciones entre el docente y los estudiantes. Por ello, en los datos cualitativos, el análisis de las respuestas obtenidas en las entrevistas individuales aplicadas a los informantes clave, se empleó, (2) la observación y diario de campo a través de la analítica de aprendizaje en el ecosistema, así como el diseño narrativo, donde el equipo investigador recaba datos sobre las experiencias de ciertas personas para describirlas y analizarlas.

\section{RESULTADOS Y DISCUSIÓN}

A continuación, la presentación de resultados se divide en dos partes: a) Análisis cuantitativo (Comunicación interdependiente, Cooperación interdependiente y Colaboración interdependiente); b) Análisis cualitativo (Interdependencia en el intercambio de recursos compartidos en la presencia cognitiva y Relaciones de interdependencia positivas entre la presencia social y el profesor).

\section{Análisis cuantitativo}

Comunicación interdependiente, está relacionado con los aspectos de interacción, cohesión del equipo y la sincronía de comunicación con herramientas digitales abiertas (ver tabla 1).

Tabla 1. Comunicación interdependiente.

\begin{tabular}{|c|c|c|c|c|c|c|c|c|c|c|c|}
\hline \multirow{2}{*}{\multicolumn{2}{|c|}{$\begin{array}{c}\begin{array}{c}\text { Comunicación } \\
\text { interdependiente }\end{array} \\
\mathrm{Fi} \\
\end{array}$}} & \multicolumn{2}{|c|}{ Siempre } & \multicolumn{2}{|c|}{$\begin{array}{c}\text { Casi } \\
\text { siempre }\end{array}$} & \multicolumn{2}{|c|}{ A veces } & \multicolumn{2}{|c|}{$\begin{array}{c}\text { Casi } \\
\text { nunca }\end{array}$} & \multicolumn{2}{|c|}{ Nunca } \\
\hline & & $\%$ & $F i$ & $\%$ & $F i$ & $\%$ & $F i$ & $\%$ & $F i$ & $\%$ & \\
\hline \multirow{3}{*}{ 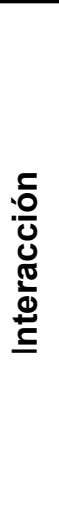 } & $\begin{array}{l}\text { 5. ¿Usted, interactúa con } \\
\text { el contenido didáctico para } \\
\text { apoyar el rendimiento de } \\
\text { todos los integrantes }\end{array}$ & 23 & 92.0 & 1 & 4.0 & 1 & 4.0 & 0 & 0.0 & 0 & 0.0 \\
\hline & $\begin{array}{l}\text { 6. ¿Usted interactúa con su } \\
\text { grupo para llevar a cabo las } \\
\text { actividades propuestas? }\end{array}$ & 24 & 96.0 & 1 & 4.0 & 0 & 0.0 & 0 & 0.0 & 0 & 0.0 \\
\hline & $\begin{array}{l}\text { 7. ¿la interacción con el } \\
\text { docente permite alcanzar el } \\
\text { logro de las metas? }\end{array}$ & 18 & 72.0 & 4 & 16.0 & 3 & 12.0 & 0 & 0.0 & 0 & 0.0 \\
\hline
\end{tabular}




\begin{tabular}{|c|c|c|c|c|c|c|c|c|c|c|c|}
\hline \multirow{5}{*}{ 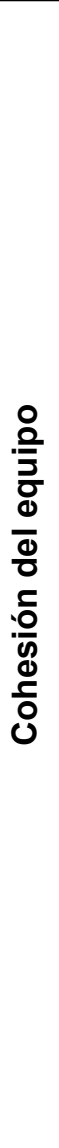 } & $\begin{array}{l}\text { 8. Usted identifica las } \\
\text { metas compartidas para } \\
\text { comunicarlas con el resto } \\
\text { del equipo? }\end{array}$ & 16 & 64.0 & 4 & 16.0 & 1 & 4.0 & 4 & 16.0 & 0 & 0.0 \\
\hline & $\begin{array}{l}\text { 9. ¿Comunica usted, con } \\
\text { los miembros del equipo el } \\
\text { compromiso para alcanzar } \\
\text { las metas compartidas? }\end{array}$ & 15 & 60.0 & 8 & 32.0 & 2 & 8.0 & 0 & 0.0 & 0 & 0.0 \\
\hline & $\begin{array}{l}\text { 10. ¿Sus compañeros } \\
\text { promueven y apoyan el } \\
\text { rendimiento del equipo? }\end{array}$ & 17 & 68.0 & 5 & 20.0 & 2 & 8.0 & 1 & 4.0 & 0 & 0.0 \\
\hline & $\begin{array}{l}\text { 11. ¿La superación } \\
\text { individual de sus actividades } \\
\text { desarrolladas, están } \\
\text { vinculadas al éxito de todos } \\
\text { los demás miembros del } \\
\text { grupo? }\end{array}$ & 14 & 56.0 & 8 & 32.0 & 1 & 4.0 & 2 & 8.0 & 0 & 0.0 \\
\hline & $\begin{array}{l}\text { 12.- ¿Su rendimiento } \\
\text { depende del esfuerzo de } \\
\text { todos los miembros del } \\
\text { equipo para alcanzar las } \\
\text { metas compartidas? }\end{array}$ & 13 & 52.0 & 9 & 36.0 & 1 & 4.0 & 2 & 8.0 & 0 & 0.0 \\
\hline 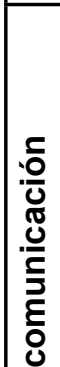 & $\begin{array}{l}\text { 13. ¿Participa usted de } \\
\text { reuniones a través de } \\
\text { herramientas abiertas de } \\
\text { comunicación síncronas } \\
\text { tanto con su docente, así } \\
\text { como con el equipo de } \\
\text { trabajo? }\end{array}$ & 22 & 88.0 & 1 & 4.0 & 1 & 4.0 & 1 & 4.0 & 0 & 0.0 \\
\hline $\begin{array}{l}\frac{0}{0} \\
\frac{\pi}{2} \\
\frac{0}{0} \\
\frac{0}{0} \\
\text { क }\end{array}$ & $\begin{array}{l}\text { 14. ¿Usted, responde con } \\
\text { prontitud a los correos y } \\
\text { mensajes a través de las } \\
\text { herramientas asíncronas } \\
\text { dispuesta por el equipo } \\
\text { para el logro de metas } \\
\text { compartidas? }\end{array}$ & 15 & 60.0 & 8 & 32.0 & 2 & 8.0 & 0 & 0.0 & 0 & 0.0 \\
\hline & Frecuencia Promedio & & 17.7 & & .9 & & 1.4 & & 1 & & \\
\hline & $\begin{array}{l}\text { Promedio en criterios de } \\
\text { análisis }\end{array}$ & & 90 & & & & 5.6 & & & .0 & \\
\hline
\end{tabular}

Teniendo en cuenta la tabla 1, para los ítems del 5 al 7 , referente a la interacción, se encontró que para el ítem 5 , el $96.0 \%$ de los encuestados, manifestaron que, siempre y casi siempre interactúa con el contenido didáctico para apoyar el rendimiento de todos los integrantes. En el caso del ítem 6, entre las alternativas siempre y casi siempre, el $100 \%$ de los docentes expresaron que interactúa con su grupo para llevar a cabo las actividades propuestas. Respecto al ítem 7 , 
solo el $88.0 \%$ entre siempre y casi siempre, la interacción con el docente permite alcanzar el logro de las metas. Al analizar estos resultados, se evidencia una interdependencia en la interacción de los participantes con respecto a la presencia tanto cognitiva, social y docente, es decir, los participantes dependen de la interacción con el material formativo, el grupo o equipo de trabajo y su mediador en la gestión del conocimiento. Por tanto, concuerda con Garrison et al., (2000), pues, se llevan a cabo una relación de interacción-comunicacióninterdependiente en la presencia social. Además, las interacciones se dan de diversas formas con vínculos humanos y no humanos, lo que coincide con Siemens (2005) quien señala que en los ecosistemas de aprendizaje se forma una interdependencia para la generación del conocimiento a través de nodos de conexión, ya sea una interdependencia con las personas o respecto de los recursos (Jonhson et al., 2007).

Por otra parte, los ítems 8 , al 12, dan cuenta sobre la cohesión del equipo de trabajo. Se verificó que en el ítem 8 , el $80.0 \%$ de los participantes consideraron que siempre y casi siempre identifican las metas compartidas para comunicarlas con el resto del equipo. Entretanto, en el ítem 9, el 92.0\% indicaron que siempre y casi siempre, los miembros del equipo comunican el compromiso del equipo para alcanzar las metas. Asimismo, en los ítems 10 y 11 , el $88.0 \%$ respectivamente indicaron que siempre y casi siempre sus compañeros promueven y apoyan el rendimiento del equipo, así como el éxito de los demás miembros del grupo está vinculado a la superación individual de cada uno en sus actividades desarrolladas. Mientras que, en el ítem 12 , el $98.0 \%$ de los participaron expresaron que el rendimiento depende del esfuerzo de todos los miembros del equipo para alcanzar las metas compartidas. De lo hallado en estos ítems, se infiere que la relación del grupo en torno a un objetivo común en el ecosistema de aprendizaje en línea, propician el desarrollo de relaciones interpersonales y la unión del grupo, según los roles de cada participante (Cuadrado et al., 2014), ya que pueden ser prácticos y eficaces para aprender social y mutuamente. Esto armoniza con Pérez (2009) quien sostiene que la construcción del conocimiento viene de un acto social y colectivo, de allí, que exista una interdependencia positiva respecto a las metas del equipo de trabajo (Jonhson et al., 2008).

Finalmente, los ítems 13 y 14 dan cuenta a la sincronía de comunicación, donde se encontró que los encuestados dijeron siempre y casi siempre $(92.0 \%)$ sobre como participan de reuniones a través de herramientas abierta de comunicación síncronas, tanto con el docente y el equipo de trabajo, así como, la disposición de responder con prontitud a través de las herramientas asíncronas (correos, móvil, foros, etc.) para el logro de metas compartidas. Esto indica que, las tecnologías de la comunicación son fundamentales en los ecosistemas de aprendizaje en línea, debido que son el medio principal de interacción de los equipos virtuales de trabajo (Rico et al., 2006).

Cooperación interdependiente, se refiere a los aspectos de responsabilidad individual, interacción promotora y habilidades sociales (ver tabla 2). 
Tabla 2. Cooperación interdependiente.

\begin{tabular}{|c|c|c|c|c|c|c|c|c|c|c|c|}
\hline \multirow{2}{*}{\multicolumn{2}{|c|}{$\begin{array}{c}\text { Cooperación } \\
\text { interdependiente } \\
F i\end{array}$}} & \multicolumn{2}{|c|}{ Siempre } & \multicolumn{2}{|c|}{ Casi siempre } & \multicolumn{2}{|c|}{ A veces } & \multicolumn{2}{|c|}{$\begin{array}{c}\text { Casi } \\
\text { nunca }\end{array}$} & \multicolumn{2}{|c|}{ Nunca } \\
\hline & & $\%$ & $\mathrm{Fi}$ & $\%$ & $\mathrm{Fi}$ & $\%$ & $F i$ & $\%$ & $\mathrm{Fi}$ & $\%$ & \\
\hline \multirow{4}{*}{ 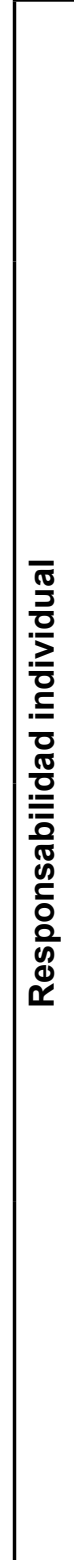 } & $\begin{array}{l}\text { 15. ¿Trabaja } \\
\text { usted en conjunto } \\
\text { con los miembros } \\
\text { del equipo para el } \\
\text { logro de metas? }\end{array}$ & 17 & 68.0 & 5 & 20.0 & 3 & 12.0 & 0 & 0.0 & 0 & 0.0 \\
\hline & $\begin{array}{l}\text { 16. ¿Coopera } \\
\text { usted en los } \\
\text { encuentros } \\
\text { grupales vía } \\
\text { móvil y reuniones } \\
\text { en línea para } \\
\text { el logro de } \\
\text { las metas del } \\
\text { equipo? }\end{array}$ & 10 & 40.0 & 8 & 32.0 & 7 & 28.0 & 0 & 0.0 & 0 & 0.0 \\
\hline & $\begin{array}{l}17 . \text { ¿Asume } \\
\text { usted, un rol } \\
\text { dentro del } \\
\text { equipo con } \\
\text { responsabilidad } \\
\text { para el logro de } \\
\text { las metas? }\end{array}$ & 17 & 68.0 & 6 & 24.0 & 2 & 8.0 & 0 & 0.0 & 0 & 0.0 \\
\hline & $\begin{array}{l}\text { 18. ¿Tiene usted } \\
\text { un compromiso } \\
\text { con el éxito } \\
\text { personal y } \\
\text { colectivo? }\end{array}$ & 12 & 48.0 & 10 & 40.0 & 3 & 12.0 & 0 & 0.0 & 0 & 0.0 \\
\hline
\end{tabular}




\begin{tabular}{|c|c|c|c|c|c|c|c|c|c|c|c|}
\hline \multirow{3}{*}{ 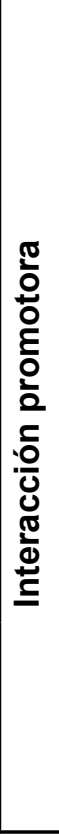 } & $\begin{array}{l}\text { 19. ¿Usted } \\
\text { anima a sus } \\
\text { compañeros de } \\
\text { equipo? }\end{array}$ & 5 & 20.0 & 5 & 20.0 & 12 & 48.0 & 3 & 12.0 & 0 & 0.0 \\
\hline & $\begin{array}{l}\text { 20. ¿Usted, sirve } \\
\text { de soporte a } \\
\text { sus compañeros } \\
\text { para que ellos } \\
\text { alcancen metas } \\
\text { individuales y } \\
\text { por ende la del } \\
\text { equipo? }\end{array}$ & 6 & 24.0 & 7 & 28.0 & 10 & 40.0 & 2 & 8.0 & 0 & 0.0 \\
\hline & $\begin{array}{l}\text { 21. Usted estudia } \\
\text { la información y } \\
\text { la distribuye entre } \\
\text { los compañeros? }\end{array}$ & 12 & 48.0 & 10 & 40.0 & 2 & 8.0 & 1 & 4.0 & 0 & 0.0 \\
\hline \multirow{5}{*}{$\begin{array}{l}\frac{0}{0} \\
\frac{0}{0} \\
\frac{\pi}{0} \\
0 \\
0 \\
0 \\
\frac{0}{0} \\
\frac{\pi}{0} \\
\frac{0}{0} \\
\frac{\pi}{\pi} \\
\frac{\pi}{1}\end{array}$} & $\begin{array}{l}\text { 22. ¿Usted es } \\
\text { capaz de definir } \\
\text { un problema } \\
\text { y proponer } \\
\text { soluciones para } \\
\text { el logro de las } \\
\text { metas? }\end{array}$ & 10 & 40.0 & 9 & 36.0 & 4 & 16.0 & 2 & 8.0 & 0 & 0.0 \\
\hline & $\begin{array}{l}\text { 23. Usted escucha } \\
\text { con atención } \\
\text { para preparar } \\
\text { los trabajos de } \\
\text { las actividades } \\
\text { asignadas }\end{array}$ & 5 & 20.0 & 5 & 20.0 & 5 & 20.0 & 10 & 40.0 & 0 & 0.0 \\
\hline & $\begin{array}{l}24 . \text { Usted es } \\
\text { empático al revisa } \\
\text { los trabajos de } \\
\text { las actividades } \\
\text { asignadas. }\end{array}$ & 4 & 16.0 & 5 & 20.0 & 7 & 28.0 & 9 & 36.0 & 0 & 0.0 \\
\hline & Promedio & & 9.8 & & & & .5 & & & & \\
\hline & $\begin{array}{l}\text { Promedio en } \\
\text { criterios de } \\
\text { análisis }\end{array}$ & \multicolumn{4}{|c|}{67.2} & & 2.0 & \multicolumn{4}{|c|}{10.8} \\
\hline
\end{tabular}

En la tabla 2, relativo a la responsabilidad individual (ítems 15 al 18), se halló que para el ítem 15, entre las alternativas siempre y casi siempre, que el $88.0 \%$ de los participantes expresaron que para el logro de las metas trabajan en conjunto con los miembros del equipo. Mientras que, en el ítem 16 , se determinó que el $72.0 \%$ de los encuestados, siempre y casi siempre, cooperan para el logro de las metas del equipo cuando se reúnen en los encuentros 
vías móvil y en línea. Entretanto, en el ítem 17, el $92.0 \%$ de los participantes siempre y casi siempre, asumen con responsabilidad su rol dentro del equipo de trabajo en pro del logro de las metas. Finalmente, en el ítem 18 , el $88.0 \%$ de los encuestados siempre y casi siempre, tienen un compromiso con el éxito personal y colectivo, el resto (12.0\%) algunas veces. En virtud de lo obtenido en los ítems 15 al 18, la mayor parte de los participantes declararon que, para el logro de las metas, superación personal y colectiva del equipo se requiere de la responsabilidad y compromiso de todos, pero especialmente de cada uno de los miembros (García y Suárez, 2011).

Por otra parte, los ítems del 19 al 21, concerniente a las interacciones promotoras, se comprobó que para el ítem 19, que el $48.0 \%$ de los participantes, algunas veces, animan a sus compañeros, un $12.0 \%$ nunca lo hace y el resto el $40.0 \%$ siempre y casi siempre, animan a sus compañeros. Asimismo, en el ítem 20, se determinó que el $52.0 \%$ de los participantes, siempre y casi siempre, sirven de soporte a sus compañeros para que ellos alcancen metas individuales $\mathrm{y}$, por ende, la del equipo; y el $40.0 \%$ algunas veces, son de soporte para sus pares. Entretanto, en el ítem 21, se conoció que el $88.0 \%$, siempre y casi siempre, estudian la información suministrada y la distribuyen entre sus compañeros. En consecuencia, se deduce que para un buen desenvolvimiento del aprendizaje en el ecosistema se requiere un proceso de comunicación de significados a partir de relaciones de mutualidad, que facilite la ayuda con recursos e influya y promueva los esfuerzos del otro para alcanzar de objetivos y/o logros de tareas interdependiente (Casanova et al., 2009).

Finalmente, en lo referente a habilidades sociales (ítems del 22 al 24) se encontró para el ítem 22, que el $76.0 \%$ de los participantes siempre y casi siempre, tienen la capacidad de definir un problema y proponer soluciones para el logro de las metas. Mientras que, en el ítem 23 , se halló que el $36.0 \%$ casi nunca escucha con atención para preparar los trabajos de las actividades asignadas, otro $28.0 \%$ algunas veces y un $10.0 \%$ siempre y casi siempre hacen en los trabajos asignados. Este resultado, está en concordancia con el ítem 24 , ya que un $9.0 \%$ son empáticos al revisar los trabajos de los otros. Por consiguiente, los resultados dan cuenta de que la cooperación se establece con interdependencia en relación con las habilidades sociales que se dan entre los componentes del grupo, y que se apoya en la comunicación y la interacción como elementos esenciales.

Colaboración interdependiente, refiere a los aspectos de participación y corresponsabilidad (ver tabla 3) 
Tabla 3. Colaboración interdependiente.

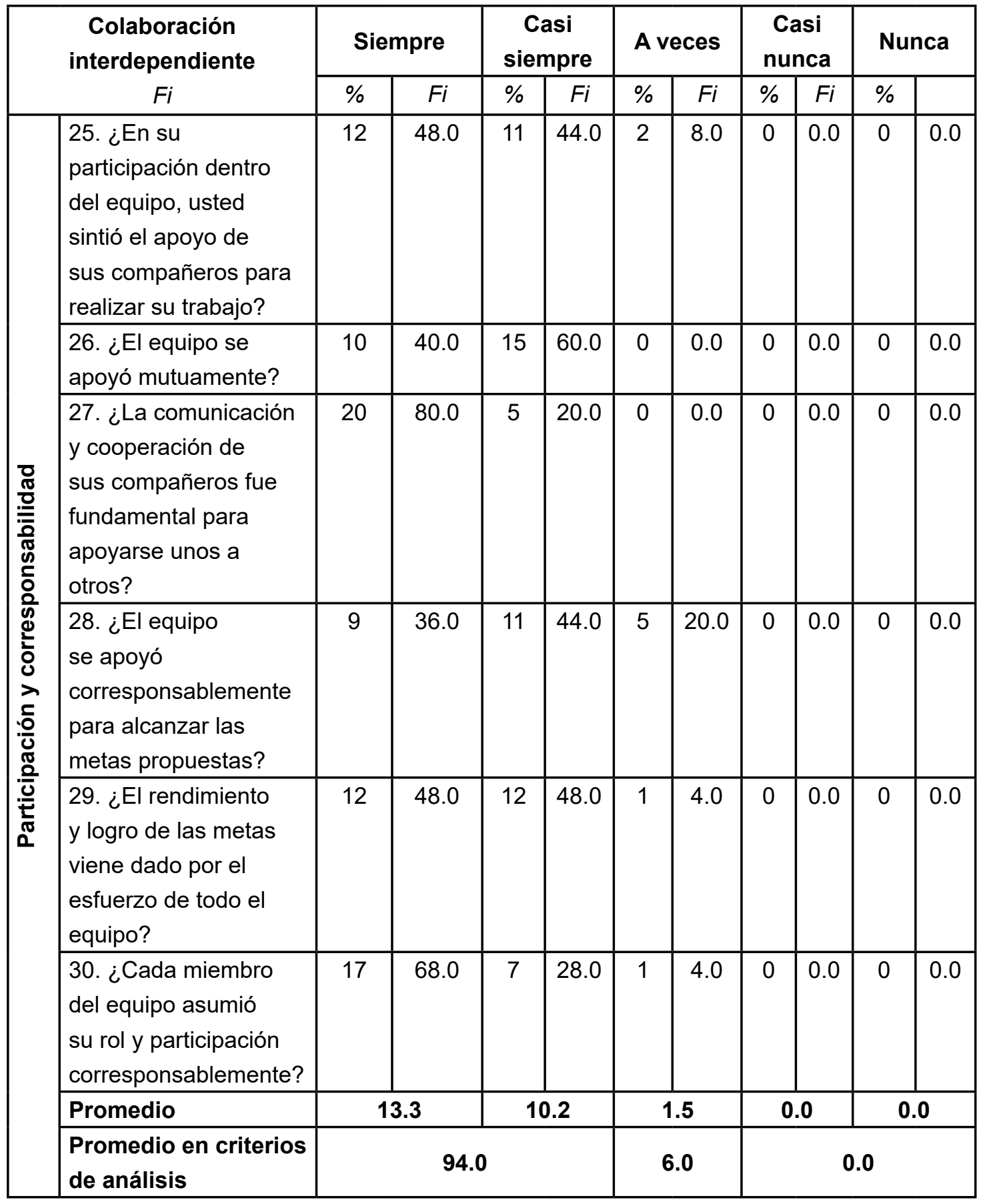

En la Tabla 3, da a conocer el análisis relativo a la participación y corresponsabilidad, donde se encontró para el ítem 25, que el $92.0 \%$ de los encuestados expresaron que, durante su participación para realizar el trabajo, siempre y casi siempre, se sintieron apoyados por sus compañeros. Mientras, que en el ítem 26, se confirmó que entre siempre y casi siempre, el
$100 \%$ se apoyó mutuamente. Entretanto, en el ítem 27, los participantes declararon que siempre y casi siempre (100\%), la comunicación y cooperación de sus compañeros fue fundamental para apoyarse. Esto coincide con el ítem 28, donde los participantes manifestaron que se apoyaron corresponsablemente para alcanzar las metas propuestas (80.0\%). 
Además, en el ítem 29 , se encontró que $96.0 \%$ de los participantes declararon que siempre y casi siempre, el rendimiento y logro de las metas viene dado por el esfuerzo de todo el equipo. Por último, en el ítem 30, se comprobó en un $96.0 \%$ que siempre y casi siempre, se debe asumir el rol y la participación en el equipo de trabajo corresponsablemente. Estos resultados (ítems 25 al 30), demuestran que la participación en el equipo se comparte la responsabilidad con otro u otros, ya que se presenta una colaboración interdependiente, donde los participantes deben asumir la responsabilidad con las metas y el éxito de su equipo, aunque sus roles, recursos y organización quedan en manos de ellos. Esto concuerda con Guitert y Pérez-Mateo (2013), quienes destacan que el trabajar juntos para alcanzar objetivos comunes en entornos virtuales se da a partir de un proceso compartido, coordinado e interdependiente en la construcción de significados y en el avance individual. De allí, se deduce que la colaboración interdependiente se caracteriza no solo por el trabajo conjunto de los participantes en grupos, sino también por el trabajo en grupo con el profesor para desarrollar conocimientos, cambiando así el entorno virtual de aprendizaje hacia un ecosistema donde la presencia social es interdependiente para una colaboración eficaz.

\section{Análisis cualitativo}

En la tabla 4, se presenta el análisis de las categorías de acuerdo con la observación directa.

Tabla 4. Observación directa de la interdependencia positiva en el ecosistema de aprendizaje

\begin{tabular}{|c|c|c|}
\hline \multirow{2}{*}{$\begin{array}{l}\text { Categorías } \\
\text { Comunicación } \\
\text { interdependiente }\end{array}$} & Observación directa & Informantes clave \\
\hline & $\begin{array}{l}\text { Entre los participantes se generó } \\
\text { interdependencia en la interacción de la } \\
\text { presencia social y el profesor de manera } \\
\text { individual y grupal para el logro de las } \\
\text { metas. } \\
\text { Al hacer analítica de aprendizaje en el } \\
\text { ecosistema se observó interdependencia } \\
\text { en el intercambio de recursos compartidos } \\
\text { en la presencia cognitiva. }\end{array}$ & $\begin{array}{l}\text { Sujeto DF: soy nueva en lo } \\
\text { virtual. Creo profundamente } \\
\text { que... desarrollamos un grupo de } \\
\text { trabajo, el cual dio sus frutos en } \\
\text { la terminación del curso, gracias } \\
\text { a VS por su paciencia y su } \\
\text { colaboración, en todos momentos } \\
\text { estuvimos comunicadas. }\end{array}$ \\
\hline & $\begin{array}{l}\text { En los foros de cada grupo se identificó que } \\
\text { las interacciones de aprendizaje dan poder } \\
\text { durante la integración, pero suelen ser } \\
\text { interacciones difíciles de mantener en las } \\
\text { relaciones interpersonales. } \\
\text { La integración en la presencia social generó } \\
\text { cohesión del grupo e interdependencia } \\
\text { positiva en la comunicación. } \\
\text { Algunos participantes proponen ideas al grupo, } \\
\text { mientras eran ayudados por sus compañeros. } \\
\text { La sincronización de la comunicación fue } \\
\text { esencial para el logro de metas. }\end{array}$ & $\begin{array}{l}\text { Sujeto VS: quiero agradecerle al } \\
\text { profesor...Se notó la presencia } \\
\text { dando respuesta a todas las } \\
\text { dudas para orientarnos en el } \\
\text { trabajo que se realizó. } \\
\text { Sujeto JCJ: este espacio se } \\
\text { ha permitido encontrarnos de } \\
\text { distintas maneras en los trabajos } \\
\text { realizados, favoreciendo una } \\
\text { conexión a veces en relación } \\
\text { con los contenidos y forma } \\
\text { de colaborar unos con otros. } \\
\text { Creo que la comunicación fue } \\
\text { primordial. }\end{array}$ \\
\hline
\end{tabular}




\begin{tabular}{|c|c|c|}
\hline $\begin{array}{l}\text { Cooperación } \\
\text { interdependiente }\end{array}$ & $\begin{array}{l}\text { Al disponer diversos recursos abierto en el } \\
\text { ecosistema, el grupo tuvo la necesidad de } \\
\text { trabajo en conjunto para cumplir con éxito las } \\
\text { metas. } \\
\text { Se estableció que los miembros del grupo } \\
\text { son interdependientes, los cuales tienden a } \\
\text { cooperar y comunicarse entre sí en mayor } \\
\text { disposición para lograr ser más productivos. } \\
\text { El grupo desarrolló una estructura en las } \\
\text { cuales se respetan las normas (hora de } \\
\text { reunión) y roles establecidos, así como } \\
\text { definieron comportamientos apropiados para } \\
\text { el grupo como un todo e individualmente } \\
\text { Los grupos eficaces desarrollanyasignan roles } \\
\text { sociales de acuerdo con los comportamientos } \\
\text { esperados. } \\
\text { Se observó que los diferentes roles a menudo } \\
\text { vienen dados con heterogéneos niveles de } \\
\text { conocimiento, poder o jerarquías. }\end{array}$ & $\begin{array}{l}\text { Sujeto GCD: sobre las temáticas } \\
\text { desarrolladas para crear el } \\
\text { estado del arte y analizar cada } \\
\text { artículo por separado, fue el } \\
\text { trabajo individual primordial } \\
\text { para luego acoplarlo de manera } \\
\text { conjunta. Sin el aporte valioso de } \\
\text { cada uno en el grupo no hubiese } \\
\text { sido posible entregar el proyecto. } \\
\text { Sujeto MDF: ... tenemos que } \\
\text { repensar las relaciones del grupo, } \\
\text { algunos compañeros se interesan } \\
\text { por apoyar a otros, mientras que } \\
\text { otros, teniendo cómo hacerlo no } \\
\text { lo hacen. }\end{array}$ \\
\hline $\begin{array}{l}\text { Colaboración } \\
\text { interdependiente }\end{array}$ & $\begin{array}{l}\text { Se observó que algunos estudiantes realizan } \\
\text { análisis muy rápidos y lanzan respuestas muy } \\
\text { aceleradas. } \\
\text { Los estudiantes se confrontan buscando dar } \\
\text { la respuesta a cada una de las preguntas. } \\
\text { Se generaron espacios para la discusión y } \\
\text { el análisis. En seudocódigo se notó un poco } \\
\text { de alarma debido al nuevo vocabulario, } \\
\text { algunos no comprenden dichos conceptos. } \\
\text { Se ayudaban entre sus compañeros para } \\
\text { entenderlos. } \\
\text { Las habilidades para la solución de problemas } \\
\text { mejoraron mediante la aplicación de todas las } \\
\text { sesiones. }\end{array}$ & $\begin{array}{l}\text { SujetoAAC: La participación de mi } \\
\text { grupo ha sido primordial en cada } \\
\text { actividad que desarrollé. También } \\
\text { me permitió un autoaprendizaje } \\
\text { del cual estoy muy agradecida a } \\
\text { todo el equipo de este curso, que } \\
\text { nos ha tenido paciencia y nos ha } \\
\text { orientado en cada proceso. }\end{array}$ \\
\hline
\end{tabular}




\begin{tabular}{|c|c|c|}
\hline $\begin{array}{l}\text { Interdependencia } \\
\text { en el ecosistema } \\
\text { de aprendizaje } \\
\text { y el logro de las } \\
\text { metas }\end{array}$ & $\begin{array}{l}\text { El enfoque conectivista en una comunidad } \\
\text { de indagación permitió el aprendizaje activo, } \\
\text { cooperativo y colaborativo en el desarrollo de } \\
\text { las actividades. } \\
\text { Por tanto, el trabajo en equipo fue fundamental } \\
\text { para el logro de las metas propuestas. } \\
\text { La interdependencia con los recursos } \\
\text { compartidos impulsó el trabajo individual, pero } \\
\text { a su vez, se fortalecieron las relaciones de } \\
\text { cooperación en el ecosistema de aprendizaje, } \\
\text { lo cual indica desarrollar estrategias } \\
\text { apropiadas para este contexto, que determinen } \\
\text { las relaciones de interdependencia. } \\
\text { Existió un grupo mínimo de estudiantes, en los } \\
\text { que su participación fue menos significativa; } \\
\text { solamente se limitaron a la presencia cognitiva, } \\
\text { con un número limitado de conexiones a los } \\
\text { recursos compartidos. }\end{array}$ & $\begin{array}{l}\text { Sujeto DF: las reuniones fueron } \\
\text { significativas ya que permitieron } \\
\text { interactuar con otras personas, } \\
\text { al mismo tiempo, compartiendo } \\
\text { diferentes puntos de vista } \\
\text { sobre el tema. Cabe resaltar } \\
\text { la importancia de trabajar en } \\
\text { grupo respetando las opiniones } \\
\text { y comentarios de los otros, muy } \\
\text { buenos aportes para nuestra } \\
\text { formación junto con todas las } \\
\text { enseñadas en este curso. } \\
\text { Sujeto AAC: ...la implementación } \\
\text { de las herramientas tecnológicas } \\
\text { facilita el compartir de } \\
\text { información, realimentando y } \\
\text { simplificando el contenido, pues } \\
\text { se adapta al horario de cada } \\
\text { estudiante para comunicarse } \\
\text { entre el grupo, así tener mayor } \\
\text { colaboración dependiendo de } \\
\text { sus posibilidades, en cuanto a la } \\
\text { cantidad de tiempo y acceso de } \\
\text { internet... }\end{array}$ \\
\hline
\end{tabular}

\section{Interdependencia en el intercambio de recursos compartidos en la presencia cognitiva}

Los hallazgos sobre el desarrollo de las actividades demandan un pensamiento profundo para relacionarse significativamente con el contenido. La interdependencia en la presencia cognitiva facilita el acceso a los recursos y materiales de aprendizaje, así como afianza la dependencia en la interacción con los docentes, las redes y la relación entre pares, pues, alcanza una positiva relación de interdependencia entre ellos. Lo que propicia la construcción colectiva del conocimiento en el ecosistema pedagógico (Castañeda y Adell, 2013). No obstante, la interdependencia con el intercambio de recursos demanda que los estudiantes no solo estén alfabetizados informacionalmente en la búsqueda de información, sino que el docente exija un nivel superior de integración en el contexto social, donde se dé lugar al cumplimiento del proceso de cognición, ya que dependen de la colaboración de los otros en la comprensión de los contenidos en línea. Ello coincide con Gutiérrez y Becerra (2014), quienes indican que los estudiantes interactúan obedeciendo de cómo los docentes estructuran la interdependencia en cada situación de aprendizaje. Como se puede apreciar, los participantes tienden a establecer una relación de dependencia entre los recursos compartidos, las actividades asignadas, el contexto social y la presencia del docente. Por ello, es necesario que, desde la presencia del docente, se aplique analítica de aprendizaje como lo enfatizan 
(Gómez-Valderrama y Ramírez-Sánchez, 2017), con el fin de orientar y mediar los procesos cognitivos y sociales dentro del grupo.

\section{Relaciones de interdependencia positivas entre la presencia social y el profesor}

Las actividades de los participantes en el ecosistema de aprendizaje ofrecen numerosas pistas que proporciona la interdependencia positiva como resultado de la superación individual y colectiva que están vinculadas al éxito de todos los demás miembros del grupo, a través de la comunicación, cooperación y colaboración. De hecho, Johnson y Johnson (2008) consideran que la interdependencia positiva es un modo determinante del trabajo grupal cooperativo. Efectivamente, los actores en red están interrelacionados y en gran medida dependen de la calidad de la conexión de la presencia social. Por otra parte, la ausencia de los participantes en las reuniones sincrónicas (presencia social), es un indicador que depende de las relaciones, en cuanto a interacción, contribución, comunicación y colaboración. También, se apreció, la relación de interdependencia en la integración de la presencia social del participante, bajo la responsabilidad individual y grupal como dimensiones esenciales para el logro de metas comunes. Ello involucra un acto social de relaciones que benefician la enseñanza y la conexión del grupo en el aprendizaje colaborativo y cooperativo. De ahí que, se da una interdependencia en cuanto a la comunicación, cooperación y colaboración siempre y cuando esté estructurada bajo la presencia del docente (coordinación) y otra no tan estructurada bajo la presencia social (integración) en conjunto con una presencia cognitiva (interdependencia de recursos compartidos) (ver figura 1).

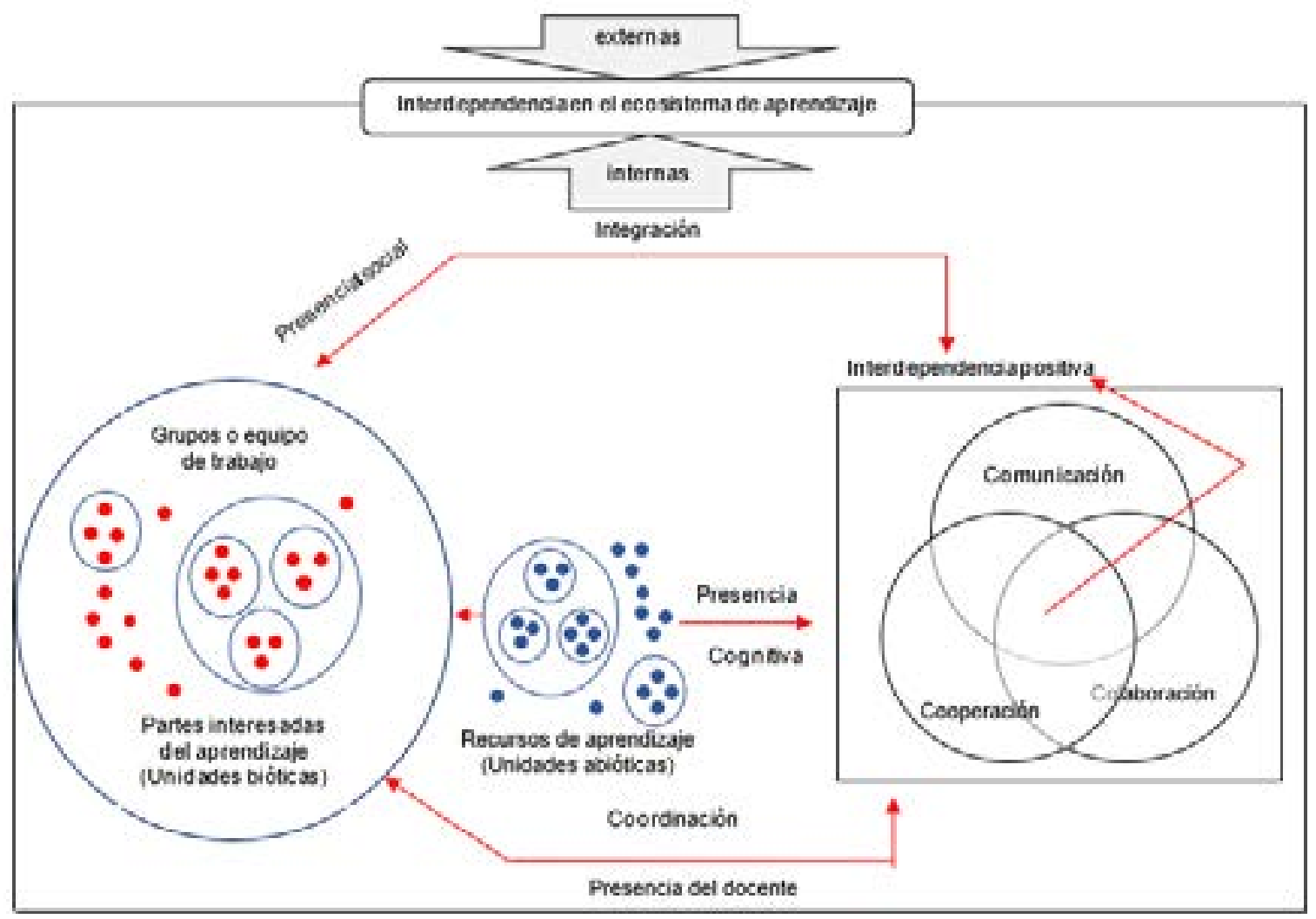

Figura 1. Interdependencia en el ecosistema de aprendizaje de una comunidad de indagación (Adaptada de Gütl y Chang, 2008; Garrison et al., 2000) 
Las deducciones de este estudio son, ciertamente, indicadores interesantes que advierten sobre el tipo de relaciones que los estudiantes hacen en el ecosistema de aprendizaje. Los hallazgos también revelaron que la interdependencia y su relación tuvieron un impacto significativo en las formasen que los participantes se integran al trabajo en grupos pequeños. Por ello, la interdependencia mutua de roles en términos de cómo interactuarán los miembros del grupo brindó el contexto a favor del aprendizaje (Suárez, 2004), aunque las conexiones de los participantes en el ecosistema tienen preferencias por algunos colaboradores por cuestiones de afinidad, que se presentan en tres (3) procesos interdependientes. Primero, el de comunicación, permite las interacciones entre individuos para compartir lineamientos, ideas $u$ opiniones bajo la presencia social del grupo. Segundo, la comunicación hace posible la cooperación; en este proceso permite la interacción en la presencia cognitiva y divulgación al compartir y agrupar recursos. Por tanto, es necesario que el ecosistema de aprendizaje fomente relaciones de comunicación/cooperación. Tercero, la coordinación de la presencia docente gestiona la comunicación y cooperación del grupo. Este proceso permite acciones de interdependencia dentro del ecosistema de aprendizaje, mediante la planificación de actividades colectivas y colaborativas, las cuales buscan un objetivo compartido (comunicación/cooperación/ colaboración) que se hallan en un aprendizaje por proyecto.

Por otro lado, lo expresado en los dos últimos apartados, dan cuenta sobre los supuestos cualitativos enunciados con anterioridad de manera afirmativa, es decir, que se cumplieron ya que el primer supuesto cualitativo, decía que una estructuración que incorpora la presencia social, presencia cognitiva y presencia del docente motivará relaciones de interdependencia positiva, permitirá el logro de las metas y objetivos propuestos en el ecosistema de aprendizaje. Mientras que, el segundo supuesto cualitativo, afirmaba que el ecosistema de aprendizaje con recursos abiertos favorece las relaciones de interdependencia positiva.

\section{CONCLUSIONES}

De acuerdo con el trabajo presentado y a los resultados obtenidos, se pueden plantear las siguientes conclusiones:

En términos de categorías, los participantes que manejaron con mayor frecuencia la comunicación con otros estudiantes en el ecosistema de aprendizaje, para llevar a cabo sus tareas fueron aquellos que tuvieron mayor participación en la presencia social y en la presencia cognitiva. En este caso, el ecosistema de aprendizaje permitió establecer relaciones tanto positivas como negativas (faltas en la comunicación y cooperación) para la transmisión, producción de conocimiento y, nodos de saberes, mostrándose interdependencia para usar y apropiarse de diferentes herramientas de comunicación.

La interdependencia, en la presencia social, presume que los esfuerzos cooperativos y colaborativos son basados en las relaciones de comunicación como primer proceso que requiere habilidades interpersonales. Por ello, el escenario de aprendizaje constituye la columna vertebral del ecosistema abierto y personal de aprendizaje, pues organiza un hilo común de comunicación asíncrona y síncrona, para los usuarios, aprendices y docentes, con lo cual se fortalecen las relaciones de interdependientes de comunicación, colaboración y cooperación real dentro de los equipos de trabajo.

Además, estas relaciones incluyen vinculaciones de afinidad entre los participantes y el docente. Cuanto más positivas sean las relaciones entre participantes y participante-docente, mayor será el compromiso y la responsabilidad 
con los objetivos del grupo puesto que son interdependiente con el éxito del otro. Es necesario comprender el ecosistema de aprendizaje y la interdependencia social, ya que pueden ser tanto positivas como negativas las relaciones de interdependencia. En este sentido, la presencia docente debe estructurar el aprendizaje cooperativo de manera efectiva; en este caso, el docente debe comprender cómo estructurar la interdependencia positiva en cuanto a la flexibilidad del ecosistema de aprendizaje.

\section{REFERENCIAS BIBLIOGRÁFICAS}

Calle, G., y Sánchez, J., Influencia de los Entornos Personales de Aprendizaje en las Habilidades Metacognitivas Asociadas a la Escritura Digital, https://doi.org/10.18041/ entramado.2017v13n1.25141, Entramado, 13(1), 158-146 (2017).

Casanova, M.O., I.M., y Gómez, I. Propuesta de Indicadores para Evaluar y Promover el Aprendizaje Colaborativo en un Debate Virtual, EDUCTEC Revista electrónica de Tecnología Educativa, (28), 1-18 (2009).

Castañeda, L. y Adell, J., Entornos Personales de Aprendizaje: Claves para el Ecosistema Educativo en Red, Marfil, Alcoy (2013)

Cívicos, A., y Hernández, M. (2007). Algunas Reflexiones y Aportaciones en Torno a los Enfoques Teóricos y Prácticos de la Investigación en Trabajo Social, https:// doi.org/10.26754/ojs_ais/ais.200723306, Acciones E Investigaciones Sociales, (23), 25-55 (2007).

Cuadrado, R., Pérez-Batlle, M., y Valero, M, Controles de Trabajo en Grupo para Mejorar la Interdependencia Positiva; en JENUI 2014 XX Jornadas de Enseñanza Universitaria de la Informática de $\mathrm{M}$. Riesco, M. Díaz, y B. López, pp 363-370, Universidad de Oviedo, Oviedo (2014)

Frielick, S., Beyond Constructivism: An Ecological Approach to E-learning; in
Beyond the comfort zone: Proceedings of the 21st ASCILITE Conference by $M$. Riesco, M.A. Díaz, y B. López, pp 328-332, ASCILITE, Perth, WA (2004).

García, Á., y Suárez, C., Interacción Virtual y Aprendizaje Cooperativo: un Estudio Cualitativo. Revista de educación, 354, 473-498 (2011)

García-Peñalvo, F.J., Ecosistemas Tecnológicos Universitarios; en UNIVERSITIC 2017. Análisis de las TIC en las Universidades Españolas de J. Gómez, pp 164-170, Crue Universidades Españolas, Madrid, España (2018).

Garrison, D., Anderson, T., y Archer, W., Critical Inquiry in a Text-based Environment: Computer Conferencing in Higher Education Model, The internet and higher education, 2(2-3), 87-105 (2000).

Gómez-Valderrama, C., Hernández-Suárez, C., y Prada-Núñez, R., La Zona de Posibilidades en el Proceso de Aprendiencia del Residente Digital: un Análisis Cualitativo en la Red de Experiencias Matemáticas de Norte de Santander, https://doi.org/10.17081/ eduhum.22.38.3688, Educación y humanismo, 22(38), 1-19 (2020).

Gómez-Valderrama, C., y Ramírez-Sánchez, R., Comunidades de Aprendizaje Móvil, Mastery Learning y el Problema 2 Sigma como Estrategias para el Conocimiento Previo, en la Resolución de Actividades, https://doi.org/10.22463/17948231.1376, Ecomatemático, 8, 25-32 (2017).

Guetl, C., y Chang, V. Ecosystem-based Theoretical Models for Learning in Environments of the 21st Century, International Journal of Emerging Technologies in Learning (IJET), 3(1), 5060 (2008).

Guitert, M., y Pérez-Mateo, M., La Colaboración en la Red: Hacia una Definición de Aprendizaje Colaborativo en Entornos Virtuales, Teoría de la Educación. Educación y Cultura en la Sociedad de la 
Información, 14(1), 10-31 (2013).

Gutiérrez, E., y Becerra, M., Los Entornos Personales de Aprendizaje (PLE). Una Experiencia de Aprendizaje Informal en la Formación Inicial del Profesorado, https:// doi.org/10.17398/1695-288X.13.2.49, Revista latinoamericana de tecnología educativa, 13(2), 49-60 (2014).

Johnson, D.W., y Johnson, R.T., Cooperation and Competition: Theory and Research, Interaction Book Company, Edina, MN (1989).

Johnson, D.W., y Johnson, R.J. Aprender juntos y solos. Edit. Aique, España (1999).

Johnson, D.W., y Johnson, R.J., Teoría de la Interdependencia Social y Aprendizaje Cooperativo: el Papel del Maestro; en El papel del maestro en la implementación del aprendizaje cooperativo en el aula. Aprendizaje colaborativo apoyado por computadora de R. Gillies, A. Ashman y J. Terwel, pp 9-37 Springer, Boston, MA (2008).

Johnson, D.W., y Johnson, R.T., Cooperative Learning in 21st Century, https://doi. org/10.6018/analesps.30.3.201241, Annals of Psychology, 30(3), 841-851 (2014).

Johnson, D.W., Johnson, R.T., y Holubec, E.J. Cooperation in the Classroom, 8a Ed., Interaction Book Co, Edina, MN (2008).

Hernández-Suárez, C.A., Perspectivas de Enseñanza en Docentes que Integran una Red de Matemáticas: Percepciones sobre la Integración de TIC y las Formas de Enseñar, https://www.doi.org/10.35575/ rvucn.n61a3, Revista Virtual universidad Católica del Norte, (61), 19-41 (2020).

Laanpere, M., Pata, K., Normak, P., y Põldoja, H., Pedagogy-driven Design of Digital Learning Ecosystems, https://doi.org/10.2298/ CSIS121204015L, Computer Science and Information Systems, 11(1), 419-442 (2014).
Marín, V., Negre, F., y Pérez, A. (2014). Entornos y Redes Personales de Aprendizaje (PLEPLN) para el Aprendizaje Colaborativo, https://doi.org/10.3916/C42-2014-03, Revista científica de educomunicación Comunicar, 42(21), 35-43 (2014).

Martínez, N., El trabajo en Equipo como Estrategia de Aprendizaje en Ambientes Virtuales, en El aprendizaje colaborativo en ambientes virtuales de L. Galindo, pp 1540, Editorial cenid, Jalisco, México (2015).

Morín, E., Introducción al pensamiento complejo, Gedisa, Barcelona (2001).

Pérez, G., La Web 2.0 y la Sociedad de la Información, Revista mexicana de ciencias políticas y sociales, 56(212), 57-68, (2011).

Pérez, M., La comunicación y la Interacción en Contextos Virtuales de Aprendizaje, Apertura, 1(1), 34-47 (2009).

Prada, R., Hernández, C., y Gamboa, A., Different Scenarios for the Teaching of Mathematics with the Support of Virtual Platforms: Flipped Classroom, J. Phys.: Conf. Se, 1388(1), 1-5 (2019).

Rico, R., Cohen, S., y Gil, F., Efectos de la Interdependencia de Tarea y la Sincronía en las Tecnologías de Comunicación sobre el Rendimiento de los Equipos Virtuales de Trabajo, Psicothema, 18(4), 743-749 (2006).

Siemens, G., Connectivism: A Learning Theory for the Digital Age. International Journal of Instructional Technology and Distance Learning, 2. 1-9 (2005).

Siemens, G., Conectivism: Learning and Knowledge Today. The International Review of Research in Open and Distance Learning, 9, 1-13 (2006). 